\title{
Análise de Usabilidade de um Sistema de EaD Baseada em Modelos Markovianos e em Taxonomia
}

Title: Usability analysis of an E-learning system based on Markovian models and in Taxonomy

Janaina R Penedo

Av. Pasteur, 458 - Urca

CEP 22290-240 - RJ - Brasil

janaina.penedo@uniriotec.br
Morganna C Diniz

Av. Pasteur, 458 - Urca

CEP 22290-240 - RJ - Brasil

morganna@uniriotec.br

\author{
Simone Bacellar Leal Ferreira \\ Av. Pasteur, 458 - Urca \\ CEP 22290-240 - RJ - Brasil \\ simone@,uniriotec.br
}

Denis Silva da Silveira

Av. dos Funcionários, s/n - Cidade Universitária

CEP: 50.740-580 - PE - Brasil

dsilveira@ufpe.br

\author{
Eliane Capra \\ Av. Pasteur, 458 - Urca \\ CEP 22290-240 - RJ - Brasil \\ eliane.capra@uniriotec.br
}

\begin{abstract}
Resumo A eficácia da Educação à Distância reside, principalmente, na interatividade entre aluno/interface/professor. Entender como o usuário se comporta, quando está conectado ao sistema, cria oportunidades para melhorias em diversas áreas, entre elas a da usabilidade. Este trabalho propõe que a análise de usabilidade de sistemas web seja feita usando a inspeção baseada em modelos Markovianos em conjunto com a inspeção baseada em taxonomia. A utilização de dois métodos permite uma avaliação mais completa da usabilidade, tendo em vista que os modelos Markovianos permitem uma avaliação quantitativa que é complementado com a avaliação qualitativa fornecida pela inspeção de taxonomia. Para verificar a viabilidade da proposta é apresentado um estudo de caso de um sistema de ensino à distância (EaD) em uso há mais de 5 anos e com mais de 60 mil alunos.
\end{abstract}

Palavras-Chave: Usabilidade Web, Modelos de Markov e Ensino à Distância.

\begin{abstract}
The effectiveness of E-learning resides mainly in the interactivity between student / interface / teacher. Understand how the user behaves when it is connected to the system, creates opportunities for improvements in several areas, including usability. This paper proposes a usability methodology for web systems based on Markovian models and taxonomy. The use of two methods allows a more complete assessment of usability, since the Markovian allow a quantitative assessment that is complemented with the qualitative assessment provided by inspection of taxonomy. A case study of an E-learning application in use for over 5 years and with more than 60 thousand students is presented to validate the proposal.
\end{abstract}

Keywords: Usability, Markov Models, E-Learning System. 


\section{Introdução}

A eficácia da Educação à Distância $(\mathrm{EaD})$ está na interatividade, na facilidade de uso e na disponibilidade que o sistema à distância proporciona a seus usuários [1]. Em um ambiente de EaD, cada participante tem a oportunidade de: fazer suas próprias escolhas entre as informações, ligar contextos, mídias e recursos; tornar-se receptor e emissor de informações, leitor, escritor e comunicador [2].

A Educação, seja a distância ou presencial, tem que ser de qualidade, não importando os meios utilizados [3]. O sistema $W e b$ disponibilizado deve favorecer a aprendizagem do aluno, provendo as informações de maneira organizada e sem erros operacionais. Os desenvolvedores não podem se esquecer do que o usuário deseja do sistema e nos efeitos que sua utilização pode produzir [4]. É importante que, ao se desenvolver sistemas para $\mathrm{EaD}$, sejam respeitadas as recomendações de usabilidade, característica que determina se o manuseio de um produto é fácil e rapidamente aprendido, dificilmente esquecido, não provoca erros operacionais, oferece um alto grau de satisfação para seus usuários, e eficientemente resolve as tarefas para as quais ele foi projetado $[5,6,7]$.

Um sistema de Educação à Distância $(\mathrm{EaD})$ é um sistema $W e b$ que integra ferramentas e serviços onde a interação aluno/computador/professor ocorre através da utilização de uma interface, parte através da qual os usuários acessam os sistemas para realizar suas tarefas e envolve todos os aspectos de um sistema com o qual o usuário mantém contato [8]. Tais sistemas devem ser desenvolvidos para disponibilizar ferramentas que permitam o ensino-aprendizagem fornecendo uma interface fácil e flexível para não desestimular o interesse dos usuários [9].

Entretanto, o desenvolvimento de qualquer sistema de informação é uma tarefa difícil por envolver não só as tecnologias como também diferentes fatores organizacionais [5]. O aprimoramento contínuo da usabilidade auxilia os usuários, independente da idade ou do grau de instrução, a conectarem-se aos mais variados sistemas, de maneira mais rápida, fácil e produtiva - reduzindo os sentimentos de frustração e impotência [6].

Problemas relacionados à navegação (usuários sentem dificuldade em encontrar a informação desejada ou não sabem como retornar as páginas anteriormente visitadas), recursos multimídia (uso de maneira inadequada devido a utilização abusiva de textos em destaque), fatores culturais (uso de referências culturais não globais) e tecnologia (incompatibilidade entre os softwares de navegação ou hardware) podem ser listados como obstáculos de usabilidade que impedem os usuários de sistemas de informação, principalmente os voltados para a prática de ensino, realizar suas tarefas [10].
Antes de liberar um sistema pronto para uso, deve ser verificado se ele apoia adequadamente os usuários nas suas tarefas e no ambiente em que será utilizado [11]. A avaliação de interface deve ser realizada para conhecer o que os usuários querem e os problemas que experimentam [7]. Quanto mais cedo forem encontrados os problemas, menor é o custo para repará-los. Logo, a análise da usabilidade pode auxiliar os desenvolvedores a melhor utilizar as tecnologias disponíveis para atender as necessidades dos usuários. Esta preocupação deve estar presente desde a fase inicial de projeto e durante todo o ciclo de vida do sistema [12].

Na realidade, a avaliação da usabilidade se refere aos métodos utilizados por avaliadores para examinar os aspectos relacionados a uma interface. Um sistema orientado para a usabilidade deve possuir uma interface onde a execução da tarefa possa ser realizada sem chamar nenhuma atenção para si, de modo que os usuários não precisem gastar sua energia na interface, mas somente no trabalho que se deseja executar [5].

De acordo com o método a ser utilizado, a avaliação da usabilidade de interfaces pode ser classificada em dois grupos [13]: métodos de observação (testes que envolvem os usuários) e métodos de inspeção (métodos analíticos, sem participação do usuário). A participação de usuários nos testes permite que se detecte problemas não tão evidentes, porém nem sempre as condições para realização de tais testes são possíveis. Por outro lado, os métodos de inspeção permitem que a avaliação transcorra sem a necessidade do envolvimento direto dos usuários [14], pois centralizam o estudo na melhoria da qualidade da interface [15]. O que é uma boa característica, já que usuários reais são difíceis e caros para serem recrutados [7].

O objetivo deste trabalho é avaliar a usabilidade de um sistema web de EAD utilizando a inspeção baseada em modelos Markovianos em conjunto com a inspeção baseada em taxonomia. Os resultados obtidos com o primeiro método são quantitativos e úteis para apresentar informações a indivíduos como empresários, gerentes de produção e administradores. Os resultados do segundo método são qualitativos e úteis para apresentar resultados a indivíduos que lidam diretamente com o desenvolvimento do produto como projetistas, analistas de sistemas e especialistas em fatores humanos. Portanto, este trabalho pretende mostrar que a integração dos dois métodos permite gerar um relatório final mais completo, facilitando assim o processo de tomada de decisão sobre alterações necessárias em um sistema Web.

Este artigo tem a seguinte organização. Na seção 2 são apresentados os conceitos usados neste trabalho e a revisão bibliográfica do tema em estudo. $\mathrm{O}$ método de pesquisa utilizado é discutido na seção 3 . A seção 4 apre- 
senta o estudo de caso, enquanto a seção 5 conclui o trabalho.

\section{Revisão Bibliográfica}

\subsection{Referencial Teórico}

Esta seção discute os conceitos relacionados à avaliação de usabilidade usando uma inspeção baseada em taxonomia e a modelos Markovianos usados neste trabalho.

\subsubsection{Avaliação de Interfaces}

A avaliação de interfaces, que permite que detectem problemas de usabilidade do sistema, consiste de um processo sistemático de coleta de dados com a finalidade de analisar como os usuários usam um artefato para executarem suas tarefas em algum ambiente computacional. Entre seus principais objetivos destacam-se: avaliar a qualidade de um projeto de interface, identificar possíveis problemas de interação, verificar conformidade a padrões entre outros [15].

Como dito anteriormente, quando os métodos de avaliação de uma interface são feitos com a presença de usuários, recebem o nome de "métodos de observação ou testes com os usuários"; quando os usuários não são envolvidos, têm-se os "métodos de inspeção ou métodos analíticos ou prognósticos". O presente trabalho aborda um método de inspeção baseado na taxonomia [16]

\section{Inspeção baseada em Taxonomia}

Os métodos de inspeção baseados em taxonomia auxiliam o avaliador no processo de identificar e de lidar com possíveis problemas no desenvolvimento de sistemas de informação, especialmente os disponíveis na $\mathrm{Web}$ [5]

A taxonomia auxilia a organização e a orientação na definição de requisitos não funcionais de usabilidade com a finalidade de sistematizar o processo de definição dos critérios de aprimoramento da usabilidade e redução de problemas. Os requisitos funcionais descrevem as funções necessárias para cumprir as tarefas no sistema. Os requisitos não funcionais (RNF) declaram os atributos de qualidade do sistema e dizem respeito a como o sistema é. Neste trabalho foi utilizada a taxonomia dos RNF de usabilidade criada por Ferreira e Leite [16] e que divide os requisitos de usabilidade em dois grupos: requisitos relacionados à exibição da informação e requisitos relacionados à entrada de dados.

\section{Requisitos relacionados à exibição da informação \\ - Consistência - Este item sugere que as telas do}

sistema devem seguir um padrão, com ícones, ações e linguagem semelhantes [17]. A consistência reduz a frustração provocada por comportamentos inesperados.

- Feedbback - Este requisito corresponde ao retorno das ações realizadas, dos resultados obtidos e principalmente da rapidez com que esta resposta é dada. Com isso, a sensação de erro diminui, melhorando a experiência do usuário [18]. O tempo de espera pelo retorno das informações solicitadas é fator crítico de usabilidade [5].

- Níveis de Habilidade e Comportamento Humano É fundamental levar em consideração o tipo de formação e o meio social dos usuários do sistema e identificar e compreender o grupo. Variações de personalidade e comportamento influem diretamente no sucesso de um sistema.

- Percepção Humana - A interface deve ser projetada para comportar os diversos tipos de usuários [19].

- Metáforas - Metáforas podem ser usadas em interfaces como forma de alavancar um conhecimento já existente do usuário; seu uso permite uma compreensão e experimentação de um tipo de coisa em termos de outro.

- Minimização da Carga de Memória - Deve-se reduzir ao mínimo o esforço exigido do usuário para memorizar as características da interface. [20].

- Eficiência no Diálogo, Movimento e Pensamentos - A apresentação do sistema deve ser coerente e proporcionar ao usuário a utilização de um modelo mental correto [5]. Em uma interface, devese levar em conta a distância entre cliques do mouse na realização de determinadas tarefas.

- Classificação Funcional dos Comandos nos Menus - Os comandos apresentados nos menus do sistema devem possuir uma classificação funcional, ou seja, as diversas ações devem ser devidamente representadas na interface, de forma que possuam uma organização eficiente para estabelecer diálogos objetivos, que não levem o usuário a percorrer desnecessariamente partes do sistema para realizar as operações que pretende.

- Manipulação Direta - A manipulação direta é caracterizada pela interação do usuário com a interface através de ações físicas. O usuário deve se sentir no controle do objeto representado.

- Exibição apenas da Informação Relevante - O usuário, ao interagir com a interface do sistema, 
deve visualizar somente as informações que lhe são relevantes naquele determinado contexto de uso. Do contrário pode se sentir confuso, não realizando as tarefas propostas.

- Uso de Rótulos, Abreviações e Mensagens Claras - Os significados de rótulos e abreviações devem ser claros para o usuário, levando-se em consideração o meio nos quais as pessoas estão inseridas.

- Uso Adequado de Janelas - As janelas dentro do sistema devem ser expostas, de modo que o usuário ao acessar várias ao mesmo tempo, possua uma visão ao menos parcial do que cada uma exibe.

- Projeto Independente da Resolução do Monitor O sistema deve ser desenvolvido para ser utilizado independente da resolução de monitor que o usuário venha a utilizar.

\section{Requisitos relacionados à entrada de dados}

- Mecanismo de ajuda - Para que o usuário possa obter informações rapidamente, devem ser disponibilizados mecanismos de ajuda que facilitem o aprendizado ao manusear o sistema, auxiliando o preenchimento na entrada de dados.

- Prevenção de erros - Um sistema bem projetado deve evitar que seus usuários possam provocar erros, provendo maneiras de evitar os erros antes que eles aconteçam.

- Tratamento de erros - Caso ocorra algum erro, o sistema deve estar preparado para corrigi-lo rapidamente, evitando perda na produtividade.

\subsubsection{Modelos Markovianos}

A utilização dos resultados de uma cadeia de Markov para a avaliação da usabilidade foi proposta em 2001 por THIMBLEBLY et al [21]. Os autores utilizaram essa técnica como forma de coleta de informações para propor uma ferramenta de avaliação de usabilidade em dispositivos como forno de microondas e telefones celulares, antes da geração de um protótipo.

Em sistemas $W e b$, muitas vezes é difícil predizer com exatidão os resultados de um processo ou experiência. No entanto, se esse processo ou experiência for repetido um grande número de vezes, é possível obter regularidades que permitem a formulação de leis matemáticas, cuja base trata dos conceitos de probabilidades e modelagem estatística. Os modelos estatísticos procuram caracterizar os processos quantitativos de uma amostra de dados. Dentre os processos estatísticos, os processos estocásticos são usados para descrever os sistemas suscetíveis à ocorrência de fenômenos que evoluem de acordo com o tem- po [22].

Dentre os processos estocásticos, os modelos de Markov merecem atenção devido à facilidade de cálculo matemático e computacional e, devido à sua propriedade de memoryless: o comportamento futuro do processo é independente do comportamento passado do processo [22].

Os processos estocásticos podem ser classificados em termos dos valores que podem assumir, assim como em termos dos instantes de tempo em que podem sofrer mudanças. A probabilidade busca avaliar a ocorrência de fenômenos ou experimentos aleatórios que podem ocorrer em um espaço amostral ou conjunto de resultados possíveis do experimento aleatório [23]. O espaço amostral pode ser finito (discreto) ou infinito (não discreto). Um espaço amostral é discreto quando seu valor for contável e é contínuo (não discreto) quando seu valor é incontável. Ao valor associado aos eventos ocorridos no espaço amostral dá-se o nome de variável aleatória ou variável estocástica. Uma variável aleatória é, portanto, uma função do espaço amostral para a qual é possível calcular a probabilidade de ocorrência de seus valores [23].

O modelo Markoviano é um modelo estocástico que possui parâmetros observados e que, apesar de não ser possível prever precisamente os valores que assumirão no futuro, gera a possibilidade de avaliar as probabilidades associadas a tais valores [24]. Eles podem ser utilizados para representar sistemas nos quais ocorrem mudanças ou transições entre estados, isto é, onde a ocorrência de uma ação marca a transição de um estado $n$ para um próximo estado $n+1$ e onde o estado anterior é irrelevante para a predição dos estados seguintes [25, 26].

A transição entre estados é feita de acordo com uma probabilidade que depende apenas do estado de origem e do estado de destino. No caso dos modelos Markovianos de tempo discreto (também chamados de cadeias de Markov), o tempo de permanência em cada estado segue uma distribuição geométrica. No caso de um processo de Markov de tempo contínuo, o tempo de permanência num estado segue uma distribuição exponencial. Um processo Markoviano pode ser representado por diagramas de fluxo, onde os nós representam os estados e os arcos representam as transições entre esses estados no tempo [27].

Considerando apenas os modelos Markovianos de tempo discreto, é possível fazer algumas observações sobre a avaliação de desempenho dos mesmos. É possível ainda estender esses conceitos para os modelos Markovianos de tempo contínuo.

No estudo de modelos Markovianos, é importante definir a distribuição de probabilidades para o estado inicial, ou seja, definir as probabilidades iniciais de cada esta- 
do do sistema (início da observação). O vetor $\pi(0)$ representa esta distribuição e é definido pela equação (1).

$$
\pi(0)=\left[\pi_{0}(0), \pi_{1}(0), \pi_{2}(0), \ldots\right]
$$

onde $\pi_{\mathrm{i}}(0)$ representa a probabilidade de o sistema estar no estado i no inicio do tempo de observação.

$\mathrm{O}$ símbolo $p_{\mathrm{ij}}$ é usado para representar a probabilidade de que, dado que o sistema está no estado $i$ em certo momento, ele passe para o estado $j$ no intervalo de tempo seguinte. Portanto, $p_{\mathrm{ij}}$ representa a probabilidade de transição do modelo de Markov do estado $i$ para o estado $j$. A possibilidade de ir de um estado $i$ para um estado $j$ é representada pela matriz de probabilidades de transição. Por exemplo, a equação (2) representa a matriz de probabilidades de transição de um sistema com $k$ estados.

$$
\boldsymbol{P}=\left|\begin{array}{cc}
P_{1,1} & P_{1,2} \ldots P_{1, \mathrm{k}} \\
P_{2,1} & P_{2,2} \ldots P_{2, \mathrm{k}} \\
P_{\mathrm{k}, 1} & P_{\mathrm{k}, 2} \ldots P_{\mathrm{k}, \mathrm{k}}
\end{array}\right|
$$

Considerando que $P$ é a matriz de probabilidades de transição entre os estados do sistema, pode-se calcular as probabilidades dos estados após $\mathrm{n}$ transições usando a equação (3) [27].

$$
\pi(n)=\pi(n-1) P
$$

Quando a cadeia é irredutível (todos os estados podem ser alcançados a partir dos demais) e o número de transições tende a infinito $(n \rightarrow \infty)$, tem-se o vetor das probabilidades estacionárias mostrada na equação (4).

$$
\pi=\pi P
$$

onde, $\pi=\left[\pi_{0}, \pi_{1}, \pi_{2}, ..\right]$. Isto significa que, após um período muito longo de observação, espera-se encontrar o sistema no estado $i$ com probabilidade $\pi_{\mathrm{i}}$.

\subsection{Trabalhos Anteriores}

Em [28], DESHPANDE e KARYPIS utilizaram uma cadeia de Markov para prever o comportamento de usuários e avaliar a usabilidade no acesso a quatro tipos de bases de dados Web: duas bases de e-commerce, dados referentes a utilização de editores de texto $W e b$ e dados de usuários que utilizam telefonia IP.

KITAJMA et al propuseram em 2005 um método para avaliar quantitativamente a usabilidade da Enciclopédia on-line Encarta [25]. Para isso, foi utilizado o método de inspeção de usabilidade conhecido como "Cognitive Walkthrough" (Percurso Cognitivo) integrado ao método de inspeção baseado em modelos de Markov.

HAREL et al apresentaram em [29] a metodologia chamada de "Sistema de Apoio a Decisão de Interfaces de Usuário" para avaliar a usabilidade de sites Web baseada nos acessos dos usuários. A metodologia utiliza a combinação de cadeias de Markov e redes baysianas para estimar e analisar as atividades dos usuários quando interagem com um site de e-commerce.

Em [30], ENGELBRECH et al utilizaram cadeias de Markov para avaliar a satisfação dos usuários de um sistema de áudio-conferência denominado "Spoken Dialog System's". A utilização de cadeias de Markov permitiu a avaliação do comportamento de diferentes usuários, levantando as características que afetam a usabilidade.

PENEDO et al apresentaram em [31] uma inspeção de usabilidade baseada em modelos Markovianos para sistemas de $\mathrm{EaD}$, utilizando-se dos critérios discutidos por GASSENFERTH et al [32]. O objetivo do trabalho foi contribuir na correta tomada de decisões relacionadas ao desenvolvimento do sistema e auxiliar nas propostas de melhoria a serem implementadas.

Outros pesquisadores, como THIMBLEBLY et al em [21], também apontaram a vantagem em realizar inspeção de usabilidade baseada em modelos Markovianos como a possibilidade de se obter métricas que fornecem comparações quantitativas para uma rápida avaliação da usabilidade de um sistema, onde o cálculo das probabilidades de transição pode ser efetuado a partir da observação das transições de estado em um sistema real.

\section{Método de Pesquisa}

Para a presente pesquisa foi realizado um estudo de caso, de um sistema de EaD, a Plataforma CEDERJ, em uso há mais de cinco anos. $\mathrm{O}$ estudo de caso, detalhado na seção 4, foi desenvolvido nas quatro etapas:

- Coleta, análise e avaliação dos dados - nesta fase foram coletados e analisados os dados referentes aos acessos dos usuários no sistema $\mathrm{EaD}$;

- Identificação das funções do sistema - nesta fase foram levantadas e identificadas as funções que o sistema disponibiliza a seus usuários, onde uma função corresponde a um conjunto 
de facilidades (serviços) oferecido no ambiente de $\mathrm{EaD}$;

- Transformação das funções do sistema em estados de uma cadeia de Markov - as funções foram mapeadas em estados de modelos Markovianos e medidas de interesse foram geradas;

- Integração e análise de resultados - Nesta etapa foi feita a integração das informações encontradas na inspeção baseada nos modelos Markovianos com a inspeção baseada em taxonomia.

\section{Estudo de Caso}

Para o desenvolvimento deste estudo de caso foram utilizadas as informações do $\log$ de acessos dos usuários da Plataforma CEDERJ [33].

\subsection{Coleta, análise e avaliação dos dados}

A Fundação CECIERJ/Consórcio CEDERJ foi oficialmente criada em janeiro de 2000 com a união da autarquia Centro de Ciências do Estado do Rio de Janeiro (CECIERJ) e do Consórcio Centro de Educação a Distância do Estado do Rio de Janeiro (CEDERJ). O Consórcio CEDERJ reúne além da Fundação CECIERJ, seis universidades públicas sediadas no Estado do Rio de Janeiro e os seus cursos alcançam mais de 60 mil pessoas/ano residentes nos 92 municípios do Estado. No total são 32 polos onde os alunos se matriculam e podem executar uma série de atividades presenciais tais como provas, defesas de trabalhos de conclusão de curso, aulas práticas em laboratórios e estágio obrigatório. Cada disciplina oferecida é supervisionada por um ou mais professores das universidades participantes do consórcio. Além disso, os alunos contam com a figura do tutor que é responsável por tirar as dúvidas sobre o conteúdo das matérias. As tutorias podem ser presenciais (o tutor atende o aluno no polo em horários pré-determinados) ou à distância - o tutor atende o aluno por meio do telefone 0800 ou do sistema de EaD [34]. A Figura 1 apresenta a tela inicial da plataforma.

A plataforma CEDERJ é o ambiente virtual de aprendizagem utilizada pelos cursos de graduação ofertados pelo consórcio e foi implementada de acordo com o documento de Referenciais da Qualidade do MEC. A plataforma CEDERJ já apresentou diversas versões e, até 2011, ela utilizava software proprietário desenvolvido pela equipe do CEDERJ. A partir de 2012, o CEDERJ passou a utilizar o sistema Moodle como ambiente de $\mathrm{EaD}$, ficando a equipe de desenvolvimento responsável pela customização dessa ferramenta. Esse estudo utiliza dados de 2010 e 2011, e, portanto, a discussão aborda as informações referentes à versão em uso na época.

Os dados utilizados neste trabalho correspondem ao log com os acessos dos usuários no primeiro semestre de 2010 e no primeiro semestre de 2011 de 10 cursos de graduação: Matemática, Ciências Biológicas, Pedagogia, Física, Computação, Administração, Química, Licenciatura em Pedagogia, História e Turismo. A Tabela 1 lista as principais informações coletadas, onde "usuário ativo" no sistema corresponde a quem se conectou na plataforma no período analisado e "sessão" corresponde ao intervalo entre o Login (entrada no sistema) e o Logout (saída do sistema) de um determinado usuário. Além disso, entende-se por "requisição" qualquer clique de mouse que solicite um serviço no sistema.

É possível verificar a partir dos dados mostrados na Tabela 1: a taxa média de crescimento na utilização da plataforma (requisições) entre 2010 e 2011 foi acima de $150 \%$; o número de usuários ativos cresceu em média $16 \%$; fevereiro, nos dois anos, foi o mês que gerou o maior número de requisições ao sistema e o que teve o maior número de usuários conectados; apesar do crescimento significativo ocorrido entre 2010 e 2011, o tempo médio das sessões permaneceu em torno de 57 minutos.

\begin{tabular}{|c|c|c|c|c|c|}
\hline & & $\begin{array}{c}\text { Usuários } \\
\text { Ativos }\end{array}$ & Sessões & Requisições & $\begin{array}{c}\text { Tempo } \\
\text { Médio } \\
\text { das } \\
\text { Sessões } \\
\end{array}$ \\
\hline \multirow[t]{2}{*}{ Janeiro } & 2010 & 12.123 & 90.728 & 428.877 & $00: 50: 04$ \\
\hline & 2011 & 16.084 & 312.488 & 1.693 .504 & $00: 59: 18$ \\
\hline \multirow[t]{2}{*}{ Fevereiro } & 2010 & 15.443 & 279.131 & 3.885 .745 & $00: 58: 20$ \\
\hline & 2011 & 17.398 & 795.372 & 11.170 .212 & $00: 62: 04$ \\
\hline \multirow[t]{2}{*}{ Março } & 2010 & 14.400 & 310.010 & 3.705 .762 & $00: 58: 59$ \\
\hline & 2011 & 15.878 & 580.618 & 7.031 .202 & 00:67:01 \\
\hline \multirow[t]{2}{*}{ Abril } & 2010 & 13.190 & 239.419 & 2.711 .179 & 00:56:05 \\
\hline & 2011 & 14.794 & 568.954 & 6.779 .254 & 00:59:07 \\
\hline \multirow[t]{2}{*}{ Maio } & 2010 & 12.666 & 295.444 & 2.680 .623 & $00: 57: 08$ \\
\hline & 2011 & 14.228 & 508.814 & 6.157 .294 & $00: 59: 54$ \\
\hline \multirow[t]{2}{*}{ Junho } & 2010 & 12.394 & 330.201 & 2.093 .895 & 00:49:02 \\
\hline & 2011 & 14.164 & 620.248 & 5.840 .514 & $00: 55: 08$ \\
\hline
\end{tabular}

Tabela 1 - Dados referentes aos cursos nos períodos analisados $(2010 / 2011)$

\subsection{Identificação das funções do sistema}

A identificação das funções do sistema foi executada na base de dados coletada na etapa anterior e a análise mostrou que somente 25 serviços (ferramentas) foram efetivamente utilizados no período selecionado para o estudo. 


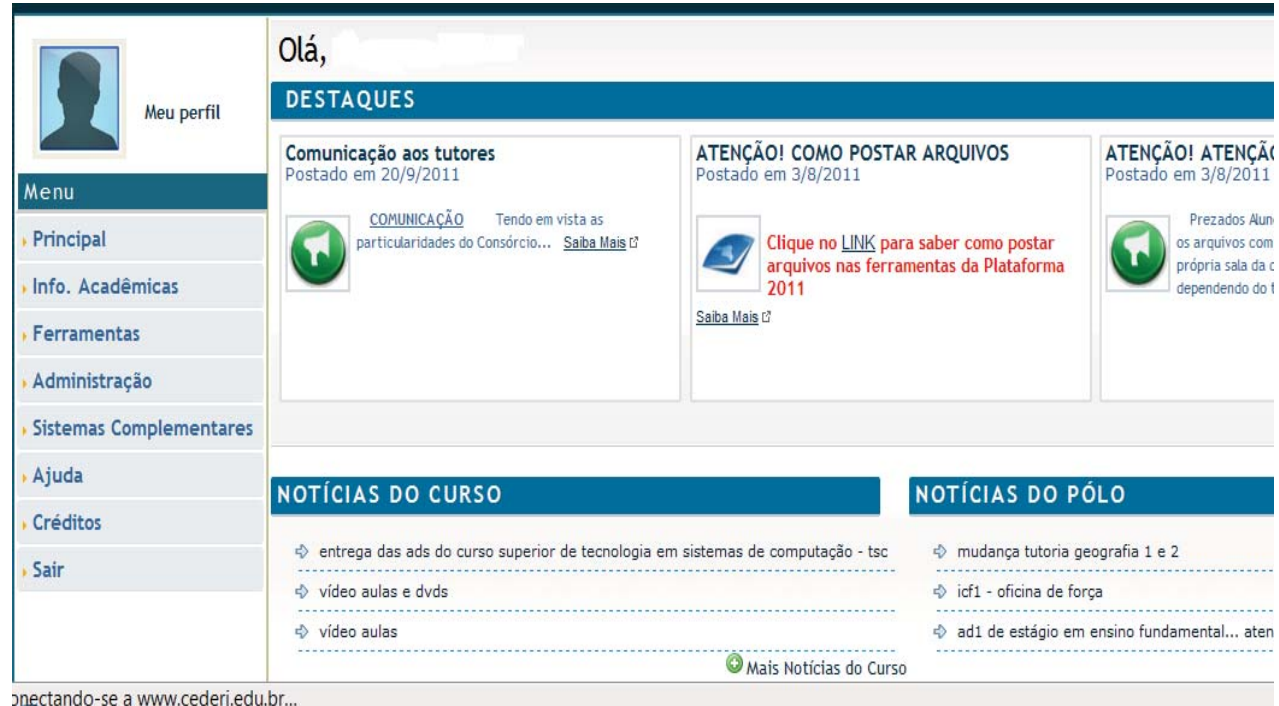

Figura 1. Tela Inicial da Plataforma CEDERJ

A Tabela 2 informa quais são estas funções.

\begin{tabular}{|c|l|}
\hline Código & \multicolumn{1}{|c|}{ Função } \\
\hline 0 & Sala de disciplina \\
\hline 6 & Perfil \\
\hline 9 & Fórum \\
\hline 13 & Sala de tutoria \\
\hline 17 & Material Complementar \\
\hline 21 & Sala de Conferência \\
\hline 29 & Gerenciador de arquivos \\
\hline 34 & Participantes \\
\hline 35 & Aviso \\
\hline 44 & E-Mail \\
\hline 46 & Mural \\
\hline 56 & Aulas na Web \\
\hline 59 & Grade de disciplina \\
\hline 75 & Calendarios \\
\hline 77 & Mural dos polos \\
\hline 78 & Mural do curso \\
\hline 83 & Atividades \\
\hline 85 & Avaliações \\
\hline 86 & Exercicios Complementares \\
\hline 89 & Tutorias Presenciais \\
\hline 92 & Blog \\
\hline 95 & Wiki \\
\hline 111 & Fale Conosco \\
\hline 123 & Material Didático \\
\hline $9999 / 33$ & Login / Logout \\
\hline & \\
\hline
\end{tabular}

Tabela 2 - Funções utilizadas em fevereiro 2010/2011.

Como observação final, nota-se que a função Login (9999) e a função Logout (33) estão agrupadas na Tabela 2. Essa função delimita o início e o fim do acesso do usuário e não indica nenhuma ação dentro do sistema. Como este estudo foca o usuário conectado à plataforma, não é necessário mapear as informações referentes ao comportamento do mesmo antes do Login e depois do
Logout

\subsection{Transformação das funções}

Considere $X$ uma cadeia de Markov de tempo discreto, com espaço de estados finito $S$ e com matriz $P$ de probabilidades de transição. Seja $P_{\mathrm{ij}}$ a probabilidade de transição do estado $i$ para o estado $j$, onde $i, j$ pertencem a $S$.

A terceira etapa do estudo consistiu em mapear as funções do sistema $\mathrm{EaD}$ utilizadas mensalmente pelos curso em estados de uma cadeia de Markov. A informação foi organizada em meses em razão da grande massa de dados existente, enquanto a organização por curso ocorreu por se querer comparar o comportamento dos alunos dos diversos cursos. Portanto, foram gerados 120 modelos, já que o estudo abrangeu dois semestres $\left(1^{\circ}\right.$ semestre de 2010 e $1^{\circ}$ semestre de 2011) e 10 cursos do CEDERJ. Cada função utilizada pelos usuários foi representada nos modelos por um estado e as probabilidades de transição foram calculadas de acordo com a Equação (5) assumindo que uma transição é um pedido qualquer de serviço pelo usuário. Em seguida, foram desenhados os diagramas de transição de alguns modelos com o objetivo de verificar visualmente o comportamento dos usuários. A partir do log dos usuários do CEDERJ, é possível calcular as probabilidades de transição usando a equação (5).

$$
P_{\mathrm{ij}}=\frac{\text { Quantidade de transições do estado } i \text { para o estado } j}{\text { Quantidade total de transições a partir do estado } i}
$$


O diagrama de transição é a representação gráfica de uma cadeia de Markov. No diagrama são visualizados os estados (representado por círculos), as transições (representadas por arcos) e as probabilidades das transições. Por exemplo, o diagrama (parcial) do curso de Matemática no mês de fevereiro de 2011 é mostrado na Figura 2. Ele representa o comportamento do aluno a partir da sua entrada no sistema (Login): $54 \%$ dos alunos vão para a Sala de Disciplina, $12 \%$ vão para a Sala de Tutoria, 5\% acessam o Aviso e 5\% acessam o e-Mail. É interessante observar que a análise dos diagramas dos outros meses mostrou que este é o comportamento padrão dos alunos da Matemática, pois as probabilidades de transição dos outros meses não apresentam grandes diferenças.

$\mathrm{Na}$ análise dos diagramas de transição dos demais cursos, foi possível observar que o aluno do CEDERJ faz uso de quatro ou cinco serviços a cada acesso ao sistema e que estes serviços normalmente são os mesmos em cada acesso. Embora o sistema de EaD do CEDERJ forneça a seus usuários um conjunto abrangente de ferramentas, o aluno utiliza apenas um pequeno número delas. Este tipo de informação pode auxiliar na melhoraria do sistema, pois permite que o desenvolvedor planeje a interface de forma que o usuário possa realizar as suas tarefas com um menor nível de interação com o sistema, melhorando assim a usabilidade.

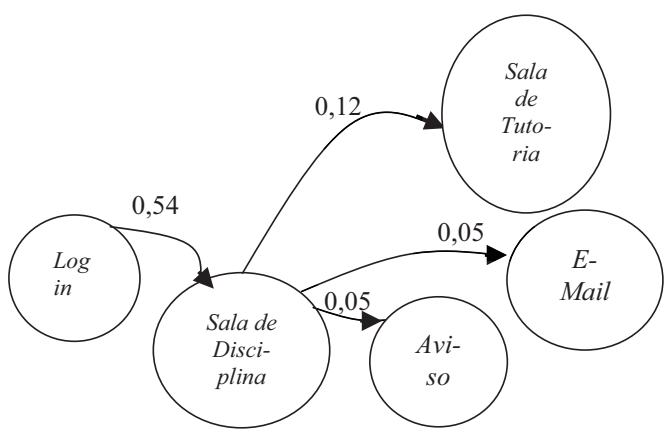

Figura 2. Diagrama de Transição referente ao curso de Matemática - fevereiro 2011

Analisando diretamente o site da plataforma CEDERJ, é possível entender o porquê dessas probabilidades de acesso iniciais. Se um usuário quiser obter qualquer informação sobre provas, avisos, gabaritos, ele irá inicialmente à Sala da Disciplina. Isto ocorre devido ao fato de algumas funções como, por exemplo, Atividades, Avaliações e Gabaritos, Exercicios e Complementos, estarem disponíveis para acesso somente após o usuário se encontrar na Sala de Disciplina, conforme mostra Figura 3 .

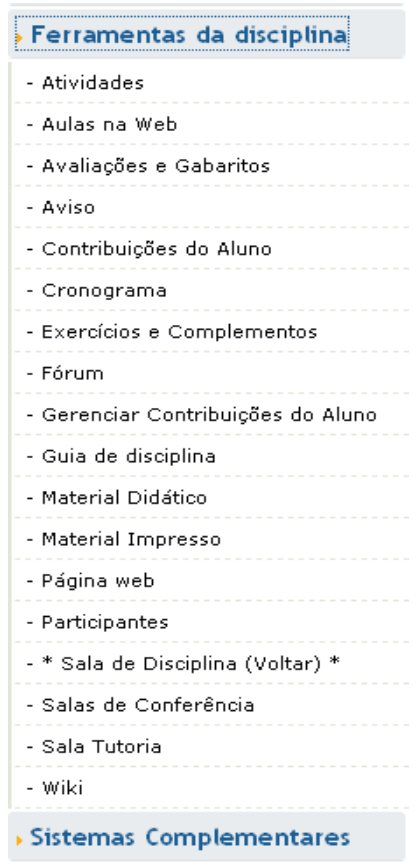

Figura 3. Ferramentas Disponíveis ao Usuário no Estado Sala de Disciplina

Dentre as funções disponíveis aos usuários após o login e que podem ser acessadas diretamente (não sendo necessário o ingresso na sala de disciplina), é possível listar: Perfil, Sala de tutorias, Blog, Material Complementar, Sala de conferência, Gerenciador de Arquivos, Mural dos cursos, Mural dos polos, E-mail e Fale conosco (Figura 4).

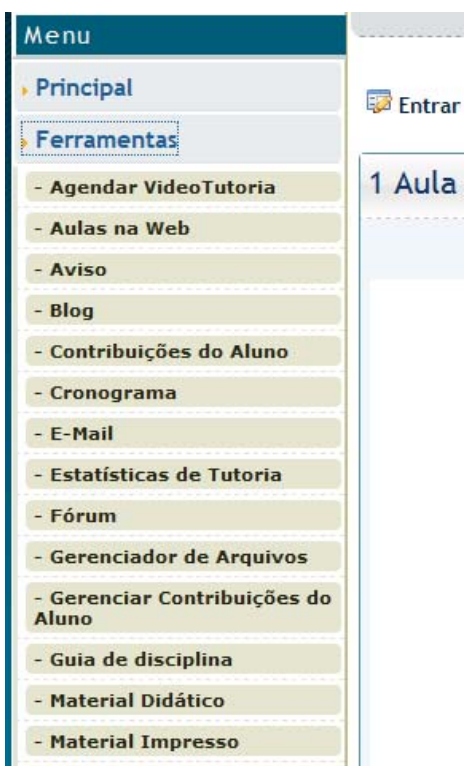

Figura 4. Ferramentas disponíveis após o login sem ser necessario ingresso na Sala de Disciplina 
As probabilidades de transição mostram ainda que, após o primeiro acesso (após a realização do Login) o usuário, não importando o curso ao qual esteja matriculado, acessa a função Sala de Disciplina com uma frequência superior a $50 \%$. Esse comportamento pode representar uma falha de usabilidade relacionada ao modo como as funções estão sendo disponibilizadas na plataforma

Também foi observado no estudo dos diagramas de transição que uma parcela considerável de alunos (acima de $10 \%$ ) sai da plataforma CEDERJ logo após efetuar o Login, sem fazer qualquer interação com o sistema (pedir algum serviço). Isso pode indicar, por exemplo, uma falha no sistema (não necessariamente de usabilidade) que merece ser investigado.

A partir das matrizes de probabilidades de transição, foi possível calcular as probabilidades estacionárias usando a equação (4). A análise mostrou que o usuário do sistema passa, no mínimo, $52 \%$ do seu tempo no sistema utilizando a função Sala de Disciplina, independentemente do curso ao qual esteja matriculado.

A Figura 5 mostra as probabilidades estacionárias de todos os cursos em fevereiro de 2011. É possível notar que cursos como Administração e Ciências Biológicas utilizam um pouco mais a função Fórum (média de 12\% do tempo de acesso) e a função Atividades (média de $10 \%$ ) que os demais cursos. O curso de Pedagogia é o que mais utiliza a função E-mail no sistema. Funções como Aulas na Web e Calendários possuem baixa utilização (2\%). Dos 57 minutos que o usuário do CEDERJ passa em média conectado, pelo menos 31 minutos foram utilizados na Sala de Disciplina.

O usuário do sistema CEDERJ ficou em média 60 minutos conectado ao sistema por sessão efetuada. Em média, nesses 60 minutos, ele passou aproximadamente 31 minutos utilizando a Sala de Disciplina. O tempo do usuário, de acordo com o curso acessado, ficou dividido da seguinte maneira:

- $\quad$ Matemática - 37 minutos do acesso utilizando a função de Sala de Disciplina, 6 minutos utiliza a Sala de Tutoria, 4 minutos acessa as demais funções;

- C. Biológicas - 30 minutos na Sala de Disciplina, 7 minutos na Sala de Tutoria, 6 minutos na função Forum, 4 minutos na função Atividades e 1 minuto nas demais funções.
- Pedagogia - 31 minutos na Sala de Disciplina, 11 minutos na função E-Mail, 6 minutos na Sala de Tutoria e menos de 1 minuto nas demais funções.

- Física - 40 minutos na Sala de Disciplina, 6 minutos na Sala de Tutoria e menos de 1 minuto nas demais funções.

- Computação - 37 minutos na Sala de Disciplina, 8 minutos na Sala de Tutoria, 5 minutos na função de E-mail e menos de 1 minuto nas demais funções.

- Administração - 22 minutos na Sala de Disciplina, 12 minutos na função Forum, 7 minutos na função Atividades e 2 minutos nas demais funções.

- Química - 20 minutos na Sala de Disciplina, 5 minutos na Sala de Tutoria, 4 minutos em Material Complementar e 2 minutos nas demais funções.

- Licenciatura Pedagogia - 33 minutos na Sala de Disciplina, 7 minutos em E-Mail, 5 minutos na Sala de Tutoria e 2 minutos nas demais funções.

- História - 35 minutos na Sala de Disciplina, 7 minutos na Sala de Tutoria, 6 minutos em Avisos e 2 minutos nas demais funções.

- Turismo - 30 minutos na Sala de Disciplina, 6 minutos na Sala de Tutoria, 5 minutos em EMail e 2 minutos nas demais funções.

Podemos assim verificar que o modelo de Markov fornece informações sobre o comportamento do usuário conectado à plataforma, mostrando o que o usuário utiliza e como utiliza.

Do estudo realizado neste capítulo, podemos tirar as seguintes conclusões relacionadas a usabilidade da plataforma CEDERJ:

- O tempo de uso em algumas ferramentas mostra pouca interatividade entre os alunos da plataforma e pode representar uma falha de usabilidade; 
Probabilidades Estacionárias

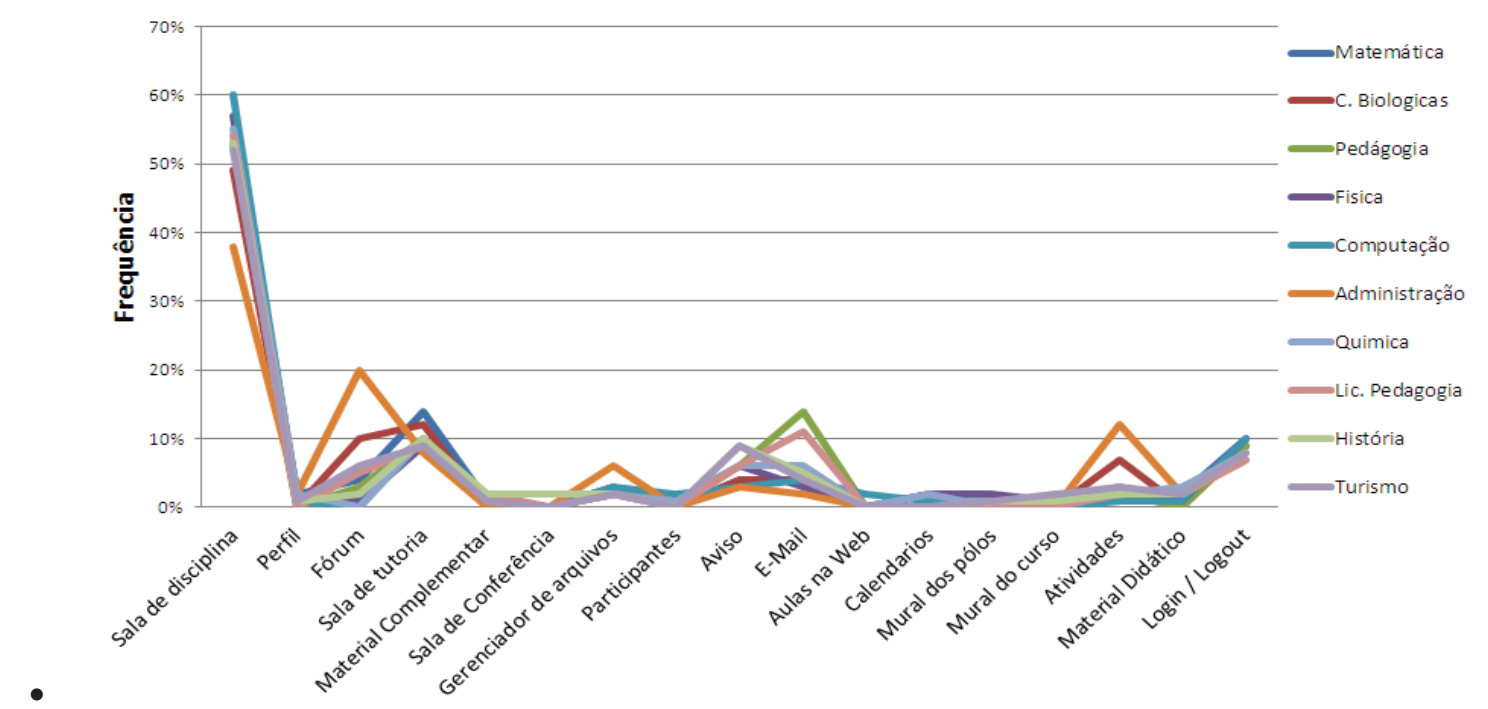

Figura 2. Probabilidades Estacionárias

- O sistema apresenta características que aumentam a produtividade e rapidez na realização de tarefas como, por exemplo, a possibilidade de a partir de uma determinada ferramenta (estado) acessar uma outra ferramenta necessária sem a necessidade de voltar a um ponto de partida, isso evita que o usuário perca tempo navegando entre menus diferentes para realizar sua tarefa;

- A partir dos diagramas do modelo Markoviano é possível verificar que somente um grupo de ferramentas é utilizado pelos usuários do sistema. Essa informação pode auxiliar em melhorias na usabilidade do sistema, aumentando a interação dos usuários com as demais ferramentas disponibilizadas;

- O modelo de Markov permite verificar o comportamento do usuário desde o momento de sua entrada no sistema (login) até o momento quando ele saí do sistema $(\operatorname{logout})$.

- As probabilidades de transição mostram que algumas ferramentas que são pouco utilizadas poderiam estar dispostas de forma a não poluir as interfaces do sistema, pois em uma única interface são apresentados todos os serviços oferecidos, mesmo aqueles poucos (ou nunca) utilizados.

Outros resultados da análise do sistema de EaD, feita a partir do modelos Markovianos, foram obtidos neste estudo. Eles não são aqui apresentados apenas por ques- tão de espaço.

\subsection{Integração e análise de resultados}

Neste trabalho foi utilizada a taxonomia dos RNF de usabilidade criada por Ferreira e Leite [23] e que divide os requisitos de usabilidade em dois grupos: requisitos relacionados à exibição da informação e requisitos relacionados à entrada de dados.

A seguir, são apresentadas as avaliações feitas a partir dos modelos Markovianos para cada um dos requisitos do método de taxonomia.

- Consistência - Com os resultados dos modelos Markovianos é possível apenas identificar comportamentos inesperados, uso de diversos nomes para uma mesma função (existência de estados absolventes - isto ocorrerá quando existirem probabilidades de transição para somente uma das funções) e uso de um mesmo nome para funções diferentes (soma das probabilidades de transição maior que 1). Neste estudo, não foram encontradas falhas referentes ao uso de diversos nomes para uma mesma função ou ao uso de um mesmo nome para funções diferentes. Com relação a comportamentos inesperados, o diagrama de transição gerado indica para quais estados o usuário pode ser encaminhado após uma determinada ação dentro do sistema. Por exemplo, foi 
detectado que algumas ferramentas estavam disponíveis somente a partir da Sala de Disciplina.

- Feedback - A partir do modelo Markoviano não é possível identificar qualquer função de feedback no estudo de caso realizado.

- Níveis de Habilidade e Comportamento Humano - O modelo Markoviano permite identificar como os usuários navegam dentro do sistema. Além disso, as probabilidades estacionárias do modelo Markoviano podem informar como os usuários gastam o tempo dentro do sistema na realização de suas tarefas.

- Percepção Humana - Com o modelo Markoviano é possível "enxergar" todos os possíveis estados do sistema.

- Metáforas - não é possível a partir dos modelos gerados no estudo de caso avaliar este item.

- Minimização da Carga de Memória - A visualização do diagrama de transição ajuda a verificar se os acessos dos usuários às funções do sistema estão bem projetadas e se auxiliam nas escolhas dos usuários.

- Eficiência no Diálogo, Movimento e Pensamentos - Com o modelo, é possível visualizar todas as funções do sistema, o que facilita determinar o caminho que o usuário precisa percorrer entre a origem e o destino.

- Classificação Funcional dos Comandos nos Menus - As ações executadas pelo usuário são realizadas através dos comandos associados às funções do sistema. O diagrama de transição permite que o desenvolvedor verifique quantos links de acesso o sistema está gerando a partir de cada estado.

- Manipulação Direta - Não é possível a partir dos modelos gerados no estudo de caso avaliar este item.

- Exibição apenas da Informação Relevante - Este item diz respeito a não poluir a interface com informações desnecessárias. O modelo de Markov facilita a visualização deste item. Por exemplo, com poucas exceções, é possível a partir de uma determinada ferramenta do sistema, alcançar qualquer outra ferramenta.
- Uso de Rótulos, Abreviações e Mensagens Claras - Não é possível a partir dos modelos gerados no estudo de caso avaliar este item.

- Uso Adequado de Janelas - Este item verifica se a interface abre desnecessariamente novas janelas. Não é possível verificar este item da taxonomia com os modelos Markovianos.

- Projeto Independente da Resolução do Monitor Não é possível a partir dos modelos gerados no estudo de caso avaliar este item.

- Mecanismo de ajuda - Os modelos Markovianos gerados não apresentam um estado que represente esse tipo de ajuda ao usuário.

- Prevenção de erros - Os diagramas dos modelos permitem visualizar o comportamento dos usuários no sistema. Isto pode ajudar a detectar e corrigir erros de usabilidade na interface.

- Tratamento de erros - Links que facilitem o retorno do usuário ao estado inicial do sistema ou que cancelem uma determinada ação do usuário são exemplos de maneiras para tratar erros de operação.

\section{Considerações Finais}

O presente trabalho, de caráter exploratório, teve como objetivo avaliar a aderência de critérios de usabilidade usando uma inspeção quantitativa baseada em modelos Markovianos em sistemas de EaD e integrando os resultados obtidos a outro método de inspeção, para gerar os resultados qualitativos da avaliação.

Antes de iniciar o trabalho foi efetuada uma investigação sobre os diversos métodos de avaliação de usabilidade e a importância da avaliação da usabilidade nos sistemas de $\mathrm{EaD}$. Um estudo bibliográfico para obter os conceitos relacionados à utilização dos modelos Markovianos também foi efetuado.

Um estudo de caso foi então feito com o $\log$ de acessos dos usuários do sistema de EaD da Fundação CECIERJ/Consórcio CEDERJ. O estudo abordou as informações referentes ao primeiro semestre do ano de 2010 e do ano de 2011. Com os resultados do modelo gerado, foram levantadas informações importantes para melhoria ou correção de possíveis falhas na usabilidade do sistema. O modelo de Markov permitiu identificar o tempo gasto pelo usuário no uso de cada ferramenta disponibilizada na plataforma, as probabilidades de transição de uma deter- 
minada ferramenta para outra, indicando com isso quais as ferramentas mais utilizadas, entender o comportamento do usuário desde o Login no sistema até a sua saída (Logout) do mesmo. Esses dados permitem que o desenvolvedor do sistema introduza melhorias referentes a usabilidades.

Com a análise do modelo de Markov foi possível gerar apenas informações quantitativas da usabilidade do sistema investigado. Para obter um resultado mais completo, optou-se por integrar as informações dos modelos Markovianos com os aspectos abordados na inspeção baseada em taxonomia apresentada por [16]. No uso do segundo método, observou-se que nem todos os itens podiam ser avaliados a partir dos resultados gerados pelos modelos Markovianos.

Dentre os itens onde foi possível realizar a avaliação pode-se destacar:

- Níveis de Habilidade e Comportamento Humano - O modelo Markoviano permite identificar como os usuários navegam dentro do sistema e como os usuários gastam o tempo dentro do sistema na realização de suas tarefas.

- Minimização da Carga de Memória - A visualização do diagrama de transição ajuda a verificar se os acessos dos usuários às funções do sistema estão bem projetadas e se auxiliam nas escolhas dos usuários.

- Classificação Funcional dos Comandos nos Menus - $\mathrm{O}$ diagrama de transição permite que o desenvolvedor verifique quantos links de acesso o sistema está gerando a partir de cada estado.

Entretanto, a integração dos dois métodos tornou possível melhorar o relatório final de usabilidade do sistema ao identificar uma maior quantidade de falhas no projeto e ao ampliar as sugestões de melhorias.

Pode-se listar como contribuições deste trabalho:

- Verificação de como os recursos disponibilizados por um sistema EaD são acessados e utilizados pelos alunos;

- Prever a necessidade de melhorias na usabilidade do sistema EaD antes que problemas comecem a surgir;

- Avaliar a aderência de critérios de usabilidade em um sistema EaD já desenvolvido e em utilização;
- Identificar o padrão de comportamento de usuários que utilizam um sistema de EaD.

\section{Referências}

[1] J. F. HERMIDA. A Educação à Distância: história, concepções e perspectivas. In Revista HISTEDBR On-line. 2006

[2] M. E. B. ALMEIDA. Distance learning on the internet: approaches and contributions from digital learning environments. In Educação e Pesquisa, São Paulo. 2003.

[3] F. NASCIMENTO, B. CARNIELli. Ensino superior: expansão com qualidade. In São Paulo em Perspectiva - SciELO Brasil. 2009.

[4] N. S. GOMES. Qualidade de Software - Uma Necessidade. Disponível em www.esaf.fazenda.gov.br/cst/arquivos/Qualidade _de_Soft.pdf. Acessado em fevereiro de 2011.

[5] S. B. L FERREIRA, R. R. NUNES. eUsabilidade. $1^{\mathrm{a}}$ edição, Rio de Janeiro, LTC Editora. 2008.

[6] J. NIELSEN, H. LORANGER. Usabilidade na web: projetando websites com qualidade, $1^{\text {a }}$ edição. Rio de Janeiro, Elsevier. 2007.

[7] H. V. ROCHA, M. C. C. BARANAUSKAS. Design e Avaliação de Interfaces HumanoComputador. Campinas (SP): NIED/Unicamp. 2003.

[8] T. MORAN. The Command Language Grammars: a representation for the user interface of interactive computer systems. In International Journal of Man-Machine Studies. 1981.

[9] C. ARDITO , M. MARSICO, R. LAZILOTTI, et al. Usability of E-learning Tools. In Proc. AVI 2004. ACM Press. 2004.

[10] L. L. GONÇALVES, M. S. PIMENTA. EditWeb: Auxiliando Professores na Autoria de Páginas Web que Respeitem Critérios de Usabilidade. In Novas Tecnologias na Educação VI No.2. Disponível em: http://www.cinted.ufrgs.br/ciclo2/resumoleila.pd f. Acesso em 25 de fevereiro de 2012.

[11] R. O. PRATES, R. M. V. FIGUEIREDO. Um Modelo de Apoio ao Projeto de interfaces e Ambientes de Aprendizado In IX WIE Workshop de Informática na Escola. Anais do XXIII SBC. 2003. 
[12] A. L. ANDRADE. Usabilidade de Interfaces web. Avaliação Heurística no Jornalismo Online. In Rio de Janeiro E-pappers. 2007.

[13] D. MODESTO, Criação de um site em JOOMLA! Orientado à usabilidade - o caso do centro de ciências exatas e tecnologia/UNIRIO. Projeto Final de Graduação. Universidade Federal do Estado do Rio de Janeiro - UNIRIO, Centro de Ciências Exatas e Tecnologia. 2009.

[14] J. E. R. QUEIROZ. Abordagem híbrida para a avaliação da usabilidade de interfaces com o usuário. Tese de Doutorado. Universidade Federal da Paraíba, Campina Grande. 2001.

[15] R. O. PRATES, S. D. J. BARBOSA. Avaliação de Interfaces de Usuário: Conceitos e Métodos. In Anais do XXIII Congresso Nacional da Sociedade Brasileira de Computação. 2003.

[16] S. B. L. FERREIRA, J. C. S. P. LEITE. Avaliação da usabilidade em sistemas de informação: o caso do sistema submarino. In RAC-Revista de Administração Contemporânea, Vol. 7, número 2, p. 115 - 136, Abril-Junho. 2003.

[17] G. AFONSO, G. ROBERTA. Grau de Usabilidade: Um Estudo de Caso do Software Tagcomércio e do Site da TAGSOFT Ltda. In Revista e-Tec. 2010.

[18] C. C. KULPA, E. T. PINHEIRO. A Influência das Cores na Usabilidade de Interfaces através do Design centrado no Comportamento Cultural do Usuário. In Perspectivas em Gestão \& Conhecimento, No. Extra 1, p. 119-136. 2011.

[19] R. S. PRESSMAN. Software Engineering: a Practitioner's Approach, 6a edição, McGrawHill. 2004.

[20] W. CYBIS. Ergonomia e Usabilidade. São Paulo: Novatec. 2007.

[21] H. THIMBLEBLY, P. CAIRNS, M. JONES. Usability analysis with Markov Models. In ACM Trans. Comput. Human Inter. 2001.

[22] R. R. C. RODRIGUES, S. V. CARVALHO. Introdução à Modelagem Markoviana e sua Aplicação em Ciências Espaciais. Disponível em:

http://www.lac.inpe.br/ELAC/arquivos/MiniCur so_09ELAC2012.pdf. Acesso em 28 de fevereiro de 2012

[23] J. F. SOARES, A. L. SIQUEIRA. Probabilidade de Variáveis Aleatórias, $1^{\mathrm{a}}$ edição, São Paulo: Edusp. 2006.
[24] C. T. HAAN. Statistical Methods in Hydrology. The Iowa State University Press, Chapter 15, p. 289-312, 1st Ed. 1997.

[25] M. KITAJMA, N. KARIVA, H. TAKAGI, Y. ZHANG. Evaluation of website usability using Markov chains and latent semantic analysis". In: IEICE Transactions on Communications. 2005.

[26] G. BOOCH. Object Oriented Analysis and Design with Applications, 2a ed. California, Addison-Wesley. 1994.

[27] L. KLEINROCK. Queueing Systems - Theory, Vol. I. Wiley-Interscience. 1975.

[28] M. DESHPANDE, G. KARYPIS. Selective Markov Models for Predicting Web-Page Accesses. In Transactions on Internet Technology . 2004.

[29] A. HAREL, R. S. KENNET, F. RUGGERI. Decision support for user interface design: usability diagnosis by time analysis of the user activity. In Proc. Computer Software and Applications Conference (COMPSAC'08). 2008.

[30] K. P. ENGELBRECH, F. GODDE, F. HARTARD, et al. Modeling user satisfaction with Hidden Markov Model. In Proc. of the SIGDIAL. 2009.

[31] J. PENEDO, M. DINIZ, S. B. L. FERREIRA, et al. Modelos de Markov aplicados na avaliação de Usabilidade de um sistema para educação à distância. In Simpósio Brasilleiro de Sistemas da Informação - SBSI. 2012.

[32] W. GASSENFERTH, R. C. SANTOS, M. A. S. MACHADO. Systems usability evaluation metrics review. In Global Business and Technology Association Conference - GBATA, Systems Usability Evaluation Metrics Review, Madri, Espan-ha. 2008.

[33] CEDERJ, 2011, Plataforma de Ensino à Distância. Disponível em: http://portal.cederj.edu.br/fundacao. Acessado em Fevereiro de 2011. 